\title{
Extracellular vesicles and amyotrophic lateral sclerosis: from misfolded protein vehicles to promising clinical biomarkers
}

\author{
Delia Gagliardi ${ }^{1} \cdot$ Nereo Bresolin ${ }^{1,2} \cdot$ Giacomo Pietro Comi $^{1,2} \cdot$ Stefania Corti ${ }^{1,2}$ (D)
}

Received: 24 April 2020 / Revised: 20 July 2020 / Accepted: 7 August 2020 / Published online: 16 August 2020

(C) The Author(s) 2020

\begin{abstract}
Extracellular vesicles (EVs) are small reservoirs of different molecules and important mediators of cell-to-cell communication. As putative vehicles of misfolded protein propagation between cells, they have drawn substantial attention in the field of amyotrophic lateral sclerosis (ALS) and other neurodegenerative disorders. Moreover, exosome-mediated non-coding RNA delivery may play a crucial role in ALS, given the relevance of RNA homeostasis in disease pathogenesis. Since EVs can enter the systemic circulation and are easily detectable in patients' biological fluids, they have generated broad interest both as diagnostic and prognostic biomarkers and as valuable tools in understanding disease pathogenesis. Here, after a brief introduction on biogenesis and functions of EVs, we aim to investigate their role in neurodegenerative disorders, especially ALS. Specifically, we focus on the main findings supporting EV-mediated protein and RNA transmission in ALS in vitro and in vivo models. Then, we provide an overview of clinical applications of EVs, summarizing the most relevant studies able to detect EVs in blood and cerebrospinal fluid (CSF) of ALS patients, underlying their potential use in aiding diagnosis and prognosis. Finally, we explore the therapeutic applications of EVs in ALS, either as targets or as vehicles of proteins, nucleic acids and molecular drugs.
\end{abstract}

Keywords Extracellular vesicles · Amyotrophic lateral sclerosis · Prion-like properties · Biomarkers · Neurodegenerative disorders $\cdot$ Therapeutics

$\begin{array}{ll}\text { Abbreviations } \\ \text { ALS } & \text { Amyotrophic Lateral sclerosis } \\ \text { ASCs } & \text { Adipose stem cells } \\ \text { BBB } & \text { Blood brain barrier } \\ \text { C9-ALS } & \text { C9orf72-related ALS } \\ \text { C9orf72 } & \text { Chromosome 9 open reading frame 72 } \\ \text { CNS } & \text { Central nervous system } \\ \text { CSF } & \text { Cerebrospinal fluid } \\ \text { CTF } & \text { C-terminal fragment } \\ \text { DPRs } & \text { Dipeptide repeat proteins } \\ \text { EVs } & \text { Extracellular vesicles } \\ \text { FTD } & \text { Frontotemporal dementia } \\ \text { FTLD } & \text { Frontotemporal lobar degeneration }\end{array}$

Stefania Corti

stcorti@yahoo.it

1 Dino Ferrari Centre, Neuroscience Section, Department of Pathophysiology and Transplantation (DEPT), University of Milan, Via Francesco Sforza 35, 20122 Milan, Italy

2 Neurology Unit, Foundation IRCCS Ca' Granda Ospedale Maggiore Policlinico, Via Francesco Sforza 35, 20122 Milan, Italy

$\begin{array}{ll}\text { FUS } & \text { Fused-in-sarcoma } \\ \text { HRE } & \text { Hexanucleotide repeat expansion } \\ \text { IL-6 } & \text { Interleukin-6 } \\ \text { iPSC } & \text { Induced pluripotent stem cell } \\ \text { lnRNAs } & \text { Long non-coding RNAs } \\ \text { miRNAs } & \text { Micro-RNAs } \\ \text { MN } & \text { Motor neuron } \\ \text { mRNAs } & \text { Messenger RNAs } \\ \text { MVBs } & \text { Multivesicular bodies } \\ \text { MVs } & \text { Microvesicles } \\ \text { NIR } & \text { Novel INHAT repressor } \\ \text { PrLD } & \text { Prion-like domain } \\ \text { pTDP-43 } & \text { Phosphorylated-TDP-43 } \\ \text { RBP } & \text { RNA-binding protein } \\ \text { ROS } & \text { Reactive oxygen species } \\ \text { sALS } & \text { Sporadic ALS } \\ \text { SOD1 } & \text { Superoxide dismutase } \\ \text { TDP-43 } & \text { TAR DNA-binding protein } 43\end{array}$




\section{Introduction}

Extracellular vesicles (EVs) are cell-derived reservoirs of different molecules, proteins and factors, providing a crucial mean of communication and material exchange between adjoining and distant cells. They have recently gained a wide relevance in the field of amyotrophic lateral sclerosis (ALS), and neurodegenerative disorders overall, both as carriers of misfolded pathological proteins and non-coding regulatory RNAs and as vehicles of interplay between neurons and glial cells within central nervous system (CNS). Moreover, since detectable in patients' biological fluids, EVs have become attractive clinical tools with wide applications in diagnosis, prognosis and therapeutics.

\section{Extracellular vesicles: biology and functions}

EVs are encapsulated particles enriched in several molecules, including membrane and cytoplasmic proteins, lipids and nucleic acids. As shuttles, they deliver molecular cargoes to recipient cells within a biological system, representing one of the most relevant mechanisms of intercellular communication both in physiological and pathological processes.

EVs may be classified according to size, biogenesis and biochemical composition and are subdivided into microvesicles (MVs) and exosomes [1]. MVs range from approximately $100-150$ to $1000 \mathrm{~nm}$ in diameter and are shed by budding of plasma membrane. Unlike exosomes, their production is mainly calcium-dependent; other factors like cellular stress and immune response may trigger MV release. Conversely, exosomes have diameters between 30 and $150 \mathrm{~nm}$, sometimes overlapping with MVs, and are generated by inward budding of endosomal multivesicular bodies (MVBs) [2]. Depending on the cargo they are delivering, MVBs can either fuse with lysosome entering the protein degradation pathway or be transported to the plasma membrane and released by exocytosis. Exosomes are then exported into the extracellular space and are able to enter the body circulation and to cross the blood brain barrier (BBB) [3]. Further, they are detectable in biofluids like cerebrospinal fluid (CSF), blood and urine $[4,5]$, so they have acquired great relevance in the study of neurological disorders.

Surrounded by a phospholipid bilayer, EVs protect molecules from degradation and enable their delivery to adjacent and distant recipient cells. Donor cell type and physiological state determine the cargo and the composition of EVs and define their primary function. First, EVs can carry biologically active mediators involved in many biological processes, aimed to maintain homeostasis [6]. For instance, morphogens act stimulating development and cellular growth and differentiation [7], molecules released by tumor cell-derived EVs provoke angiogenesis, proliferation and metastasis [8], and bioactive lipids promote cytokine secretion, which in turn may induce chemotaxis, inflammation or apoptosis. Additionally, they might function as vehicles for elimination of unnecessary materials, playing a neuroprotective role. Similarly, exosomes have been shown to deliver active enzymes to assist degradation of potential toxic proteins [9]. Further, other than transporting useful proteins, EVs enable genetic material exchange between cells. Indeed, messenger RNAs (mRNAs) can be send to the recipient cells, where they are translated into functional proteins; besides, microRNAs (miRNAs) and long non-coding RNAs (lnRNAs) delivered by EVs may regulate gene expression in recipient cells [10].

All cell types in the CNS can release EVs, including neurons, astrocytes, oligodendrocytes and microglia [11]. In vitro studies $[12,13]$ have reported the high contribution of neuronal EVs, particularly exosomes, into synaptic activity modulation, since they can regulate the neurotransmitter release and the amount of post-synaptic receptors, as well as the neuronal excitability. MVs released by microglia are mainly involved in cytokine secretion, other than enhancing excitatory transmission [14], while microglial exosomes likely play a role in immunity [15]. Conversely, exosomes released by oligodendroglia are involved in neuronal firing rate and survival, since they can induce the transcription of genes implicated in stress response [16]. Astrocytes release a variety of EVs and according to their cargoes may mediate neurogenesis and synaptogenesis, myelination and neuroprotection or immunoregulatory and anti-apoptotic effects [17]. Importantly, astrocytederived MVs contain glutamate transporters and provide to their subcellular localization [18], consistently with astrocyte function of scavengers of extracellular glutamate.

Interestingly, a longitudinal study aimed at evaluating agerelated physiological changes in plasma EVs has shown a decrease in EV concentration due to higher B-cell-mediated internalization and variation in protein composition with age [19]. Specifically, reduced levels of apoptotic markers (e.g. p-53, caspase-3) and enrichment in proteins involved in tumorigenesis and metastasis have been found in older patients, suggesting that EV characterization may provide relevant insights into paraphysiological processes like aging and potentially on age-related chronic diseases. 


\section{Extracellular vesicles in neurodegenerative disorders}

Neurodegenerative disorders have been increasingly recognized as proteinopathies, namely diseases resulting from abnormal deposition of misfolded and/or aggregated proteins. A growing body of evidence has pointed at a prion-like propagation as a possible mechanism underlying cell-to-cell protein transmission [20, 21]. According to this prion-like hypothesis, misfolded proteins can be transferred to healthy cells, induce their endogenous counterpart to misfold and lead to the amplification of these pathological seeds, prompting the onset and the progression of neurodegenerative disorders [22]. The spreading of the seeds between anatomically connected brain areas may occur through endocytosis/pinocytosis [23], tunneling nanotubes [24] and EV-mediated secretion [25].

A number of proteins, including $A \beta, \alpha$-synuclein and tau have been found to colocalize with exosomes [26-31], supporting the role of EVs as potential drivers of pathological protein transmission and consequent neurodegeneration. Conversely, exosomes delivering proteolytical enzymes active against extracellular $A \beta$ would suggest their biological role in preventing neurodegeneration [9]. Moreover, there is also counteractive evidence suggesting that pathological proteins can be transmitted as free proteins and seed propagation may occur through mechanisms other than exosome-based secretion [32].

In addition, the concept of neurodegenerative disorders as "non-cell autonomous" diseases is progressively gaining round, thus, in this context EV-mediated cell-cell communication, as well as neuronal-glial interplay, may play a crucial role in disease pathogenesis [33]. Neuroinflammation has been suggested as putative mechanism in neurodegenerative disorders. In this context, given their ability to produce immunomodulatory cytokines, glial cells, and especially microglia, are able to sense neuronal activity and to influence synaptic transmission in response to inflammatory stimuli.

Indeed, microglia-derived EVs generated in inflammatory conditions harbor different cargoes (pro-inflammatory cytokines and miR-155) compared to EVs derived from homeostatic microglia [34]. Other than inducing detrimental effects on neurons, the production of inflammatory mediators and reactive oxygen species (ROS) leads to further microglial activation, generating a vicious cycle of inflammation and neurodegeneration. In a recent study [35], MVs from inflammatory microglia were enriched in a set of miRNAs involved in regulation of key synaptic protein expression. Prolonged exposure to miR-146a-5p-containing EVs, which inhibits the expression of presynaptic synaptotagmin 1 and postsynaptic neuroligin1, induced significant dendritic spine loss and decrease in density and strength of excitatory synapses. Consistently, astrocyte-derived exosomes from AD patients display higher expression of TNF $\alpha$, IL- $1 \beta$ and IL- 6 and higher level of complement proteins respect to controls [36]. In PD, microglial-derived EVs release several pro-inflammatory molecules in response to oxidative stress, causing harmful effects on dopaminergic neurons [37].

Notably, neuroprotective effects of glial cell-derived EVs in neurodegenerative disorders have been described as well, as shown by secretion of anti-inflammatory astrocyte-derived factors in parkinsonian brains [37]. In addition, exosomes isolated by adipose stem cells (ASCs) display neuroprotective and anti-apoptotic effects on an in vitro model of ALS [38]. Taken together, these findings support a dual role for EV-mediated glial-neuron crosstalk in neurodegenerative disorders.

Therefore EVs, particularly exosomes, have generated significant interest in the field of neurodegenerative disorders, given their application both in understanding disease pathogenesis and in improving diagnosis, prognosis and therapeutics of neurologic disorders.

ALS is a devastating disorder due to degeneration of upper and lower motor neurons (MNs). The pathological hallmark underlying most of ALS cases and half of the cases of frontotemporal lobar degeneration (FTLD) is the deposition of nuclear and cytoplasmic inclusions of TAR DNA-binding protein 43 (TDP-43) and phosphorylatedTDP-43 (pTDP-43) [39]. ALS-affected patients experienced focal weakness and atrophy, with subsequent clinical involvement of neighboring districts. The spreading of symptomatology to contiguous regions seems to trace clinically the transmission of misfolded proteins occurring on a molecular level between adjoining cells and within interconnected CNS areas.

Here, we aim to investigate the main functions of EVs in ALS, highlighting their role in disease pathogenesis. In particular, we summarize in vitro and in vivo evidence of EV-mediated prion-like misfolded protein propagation in ALS, focusing on the proteins encoded by the main ALS-causative genes, i.e. superoxide dismutase 1 (SOD1), TARDBP, Fused-in-sarcoma (FUS) and Chromosome 9 open reading frame 72 (C9orf72). Then, since alteration of RNA homeostasis is a crucial process in ALS, we provide an overview on EV-mediated non-coding RNA transmission and we point out the contribution of miRNAs into the regulation of gene expression in ALS. Finally, we explore the various applications of EVs in the field of motor neuron disorders and we underline their relevance as tools for pathogenesis comprehension, biomarkers in disease diagnosis and prognosis, as well as potential vehicles or targets for therapeutic approaches. 


\section{Evidence of prion-like EV-mediated misfolded protein propagation in amyotrophic lateral sclerosis}

Several proteins encoded by genes causative of ALS and involved in disease pathogenesis have been characterized over the past decades. Recent evidence suggests that many of these proteins have been retrieved in EVs and travel between neuronal and glial cells and within different brain areas, contributing to disease spreading and propagation [40]. Among them, particular mention has to be done to SOD1, TDP-43, FUS and dipeptide-repeat proteins (DPRs) (Table 1).

SOD1 is a cytosolic and mitochondrial enzyme involved in clearance of superoxide molecules. Misfolded SOD1 aggregates have been reported in spinal MNs and glial cells of familial ALS (fALS) cases harboring SOD1 mutations and of some sporadic forms (sALS) [41, 42]. Interestingly, SOD1 has been the first ALS-associated protein retrieved in EVs. In particular, SOD1 has been found in association with exosomes derived from mouse motor neuron-like NSC-34 cells overexpressing wild-type and mutated SOD1 [43]. The authors speculate about the possible protective role of SOD1-positive exosomes against the physiological production of ROS outside the plasma membrane. A few years later, SOD1 overexpressing astrocytes have been shown to release mutant SOD1-containing exosomes able to prompt MN death [44], thus suggesting that astrocytes and EVs may contribute to neuronal toxicity and disease spreading. After that, seed conversion of wild-type SOD1 by its misfolded counterpart has been demonstrated in intracellular compartments [45, 46], Grad and colleagues have provided evidence that misfolded native and mutated SOD1 can be transmitted from cell to cell through exosome-dependent and independent fashion

Table 1 Proteins with relevance for ALS identified in extracellular vesicles from in vitro and in vivo models and ALS patients

\begin{tabular}{|c|c|c|c|c|}
\hline Protein & Study & Extracellular vesicles & Sample/model & Main finding \\
\hline \multicolumn{5}{|c|}{ In vitro and in vivo studies } \\
\hline SOD1 & [43] & Exosomes & Mouse MN-like NSC-34 cells & $\begin{array}{l}\text { Possible protective role of SOD1-containing } \\
\text { exosomes against ROS }\end{array}$ \\
\hline SOD1 & {$[44]$} & Exosomes & SOD1 overexpressing astrocytes & $\begin{array}{l}\text { Astrocyte-derived exosomes contribute to neu- } \\
\text { ronal toxicity }\end{array}$ \\
\hline SOD1 & {$[47]$} & Exosomes & Mouse MN-like NSC-34 cells & $\begin{array}{l}\text { SOD1 is transmitted from cell to cell through } \\
\text { exosomes and misfolding native SOD1 is } \\
\text { efficiently perpetuated in naïve cells }\end{array}$ \\
\hline SOD1 & [48] & Exosomes & Rat microglial cells & $\begin{array}{l}\text { Microglial cells release SOD1-containing } \\
\text { exosomes and are toxic to neurons }\end{array}$ \\
\hline SOD1 & [49] & Exosomes and MVs & SOD1 transgenic mouse & $\begin{array}{l}\text { SOD1 is secreted in vivo in EVs derived from } \\
\text { astrocytes and neurons }\end{array}$ \\
\hline TDP-43 & {$[50]$} & Exosomes & Human neuroblastoma cells & $\begin{array}{l}\text { Phosphorylated TDP- } 43 \text { aggregates can propa- } \\
\text { gate from cell-to-cell via exosomes }\end{array}$ \\
\hline TDP-43 & {$[53]$} & Exosomes & U251 cells & $\begin{array}{l}\text { TDP-43-containing exosomes from CSF from } \\
\text { ALS/FTD patients has prion-like transmissible } \\
\text { properties in vitro }\end{array}$ \\
\hline TDP-43 & {$[55]$} & Exosomes and MVs & HEK-293 cells and primary mouse neurons & Intracellular transmission and seeding properties \\
\hline TDP-43 & {$[56]$} & Exosomes & Neuro2a cells and TDP-43 transgenic mouse & $\begin{array}{l}\text { Cytoplasmic TDP-43 localization in vitro; } \\
\text { possible contribution in TDP-43 neuronal } \\
\text { clearance in vivo }\end{array}$ \\
\hline FUS & {$[58]$} & Exosomes & SH-SY5Y and N2A cells & FUS secretion in $F U S$-overexpressing cells \\
\hline DPRs & {$[61]$} & Exosomes & $\begin{array}{l}\text { iPSC-derived MNs from C9orf72-related ALS } \\
\text { patients }\end{array}$ & Cell-to-cell DPR transmission \\
\hline \multicolumn{5}{|c|}{ Human studies } \\
\hline $\begin{array}{l}\text { SOD1, } \\
\text { TDP-43 } \\
\text { and FUS }\end{array}$ & [82] & MVs & Plasma of sALS patients & Elevated levels in ALS compared to controls \\
\hline TDP-43 & {$[54]$} & Exosomes & CSF of ALS patients & $\begin{array}{l}\text { CSF brain-derived exosomes contain TDP-43, } \\
\text { reflecting neuropathology }\end{array}$ \\
\hline
\end{tabular}

$A L S$ amyotrophic lateral sclerosis, $C S F$ cerebrospinal fluid, $D P R s$ dipeptide-repeat proteins, EVs extracellular vesicles, FTD frontotemporal dementia, FUS fused-in-sarcoma, iPSC induced pluripotent stem cell, $M N$ motor neuron, $M V s$ microvesicles, $R O S$ reactive oxygen species, $S A L S$ sporadic ALS, SOD1 superoxide dismutase, TDP-43 TAR DNA-binding protein 43 
and that propagated misfolding of native SOD1 may be efficiently perpetuated into naïve cells [47]. Further, cell exposure to conditioned media derived from SOD1 overexpressing HEK293 cells induces intracellular accumulation of misfolded SOD1, while this does not occur after conditioned media depletion from EVs [47]. SOD1 overexpressing microglial cells have been found to release SOD1-containing exosomes as well [48]. Besides, they have shown toxicity in co-culture with primary neurons, which can be alleviated by treatment with the autophagy-inducer trehalose [48]. These findings strongly support the role of EVs in misfolded protein propagation following a prion-like fashion. Recently, a study performed in SODI transgenic mouse model has shown that misfolded SOD1 protein is enriched in EVs released by neurons and astrocytes [49].

$T A R D B P$ encodes for mainly nuclear RNA-binding protein (RBP) involved in splicing and RNA metabolism. Aggregates of phosphorylated and ubiquitinated TDP-43 are the main neuropathological findings in brains from ALS patients [39]. C-terminal fragment (CTF) of TDP-43 contains a prion-like domain (PrLD) which allows protein-protein interaction and is able to induce seed-dependent aggregation in vitro [50]. Most of TARDBP disease-causing mutations involve CTF [51] and induce TDP-43 cytoplasmic mislocalization and pathological aggregate formation [52], resulting in RBP aberrant sequestration and impairment of RNA metabolism and proteostasis.

Exposure of $T D P-43$-expressing human neuroblastoma cells to insoluble TDP-43 from brains of ALS and frontotemporal dementia (FTD) patients results in template aggregation; moreover, pTDP-43 aggregates can be propagated from cell to cell at least partly via exosomes [50]. TDP-43 enrichment in EV fraction of CSF from ALS/FTD patients $[53,54]$ also supports the role of EVs in disease propagation. Moreover, CSF enriched in TDP-43-containing exosomes may act as seed in vitro, since it is able to prompt accumulation of toxic TDP-43 CTF in cell lysates [53]. Further and definitive evidence of exosome-mediated secretion and prion-like propagation of TDP-43 aggregates has emerged from the study of Feiler and colleagues [55]. Indeed, exosomes and MVs containing TDP-43 oligomers have shown intercellularly transmission, as well as seeding properties [55]. Finally, Iguchi and colleagues demonstrated that exosomal TDP-43 secretion is upregulated in brains from ALS patients and may induce cytoplasmic redistribution of TDP-43 in Neuro2a cells [56]. However, inhibition of exosome secretion in vivo increases TDP-43 aggregation and exacerbates the phenotype of TDP-43 transgenic mice, suggesting that exosomes may also be implied in neuronal clearance of TDP-43 [56].

Mutations in FUS are responsible of a subset of cases of familial and sporadic ALS. Similarly to TDP-43, FUS encodes for a RBP and its mutations may prompt protein cytoplasmic mislocalization with subsequent sequestration of RNA transcripts and stress granules-like structure formation [57]. FUS immunoreactive inclusions have been found in neuronal and glial cells of patients affected by FUSrelated fALS [57], suggesting that FUS abnormal deposition may exert neuronal toxicity and death, similarly to TDP-43 and SOD1. Recent evidence has proven that FUS colocalize with exosomes derived from wild-type and mutated FUSexpressing cells [58], possibly indicating that EVs might participate in cell-to-cell FUS propagation.

Hexanucleotide repeat expansion (HRE) in C9orf 72 are the most common genetic cause of both fALS and sALS. Along with inclusions of phosphorylated and ubiquitinated TDP-43, post-mortem tissues from patients with C9orf72related ALS (C9-ALS) have shown the presence of DPRs derived from non-canonical translation of sense and antisense HRE-containing transcripts [59]. DPR-mediated toxicity is crucial for C9-ALS pathogenesis and is considered one of the major drivers of neuronal death [60]. DPR intercellular transmission via exosome-dependent and independent pathways has been demonstrated in spinal MNs from C9-ALS patients and DPRs transfected NSC-34 cells [61]. Although causality with neuronal death has not been reported, DPRs have been found to propagate from neurons to glial cells and viceversa, likely accounting for pathology propagation in C9-ALS [61].

Taken together, these findings advocate the crucial contribution of EVs into the spreading of toxic proteins across CNS, and consequently in neurodegeneration (Fig. 1).

\section{Non-coding RNAs and EVs in ALS}

As mentioned earlier, nucleic acids are one of the principal cargoes retrieved in EVs, in addition to proteins. The phospholipid bilayer surrounding EVs guarantees RNA stability in extracellular space and in biofluids, preventing degradation by ribonucleases. Other than mRNAs, transmission of non-coding RNAs provides regulation of gene expression by promoting or inhibiting translation in recipient cells, thereby providing us with valuable insight into cell functioning and disease pathogenesis.

This is particularly relevant in ALS, given that alteration in RNA homeostasis is a well-recognized pathomechanism. Indeed, some ALS-causing genes (e.g. TARDBP and FUS) are involved in RNA processing and activity and some miRNAs may alter the expression of proteins involved in ALS. Moreover, the depletion of Dicer, an enzyme crucial for miRNA biogenesis, leads to MN death [62]. Accordingly, non-coding RNAs and especially miRNAs have become promising tools useful to better understand disease pathogenesis $[63,64]$. 


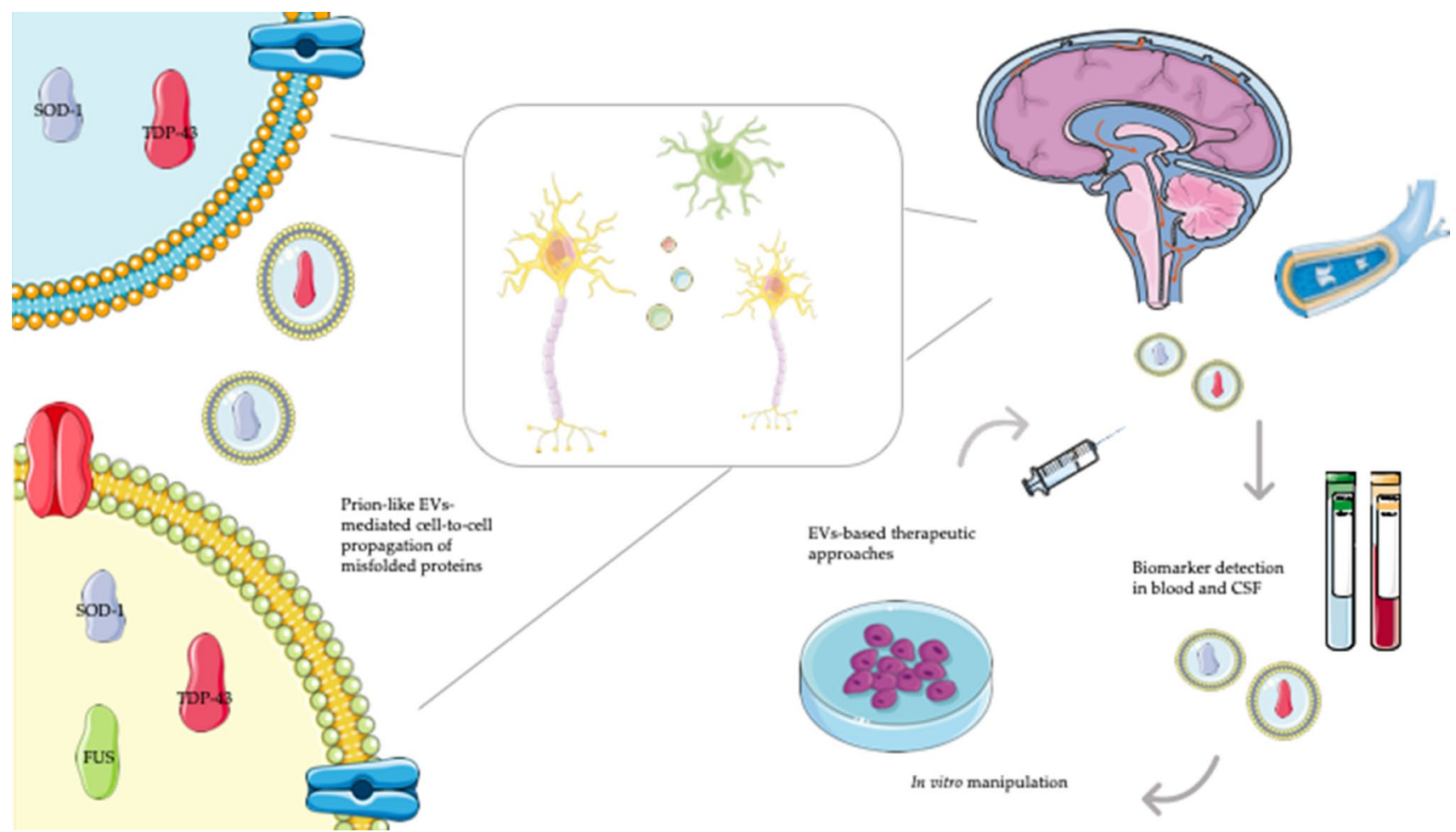

Fig. 1 Extracellular vesicle cell transmission and their clinical applications in amyotrophic lateral sclerosis. Extracellular vesicles (EVs) mediate cell-to-cell transmission of misfolded proteins (e.g. SOD1, TDP-43 and FUS) in the central nervous system (CNS). EVs can be detectable in blood and cerebrospinal fluid (CSF) of amyotrophic

A few studies have successfully elucidated the role of miRNAs carried by EVs in modulating ALS phenotype and pathogenesis.

After previous evidence of exosome-mediated SOD1 cell-to-cell transmission as mechanism of disease spreading, Pinto and colleagues have shown that wild-type and mutated SOD1 transfected NSC-34 MNs are able to transfer miRNAenriched exosomes to $\mathrm{N} 9$ microglial cells, thus influencing their inflammatory properties [65]. Indeed, increased expression of miR-124 has been found in SOD1-mutated MNs and in their derived exosomes and reduction of microglial phagocytic activity, persistent Nf-KB activation, as well as upregulation of genes involved in inflammation in microglia has been observed [65]. Moreover, miR-124 has been previously correlated to astrocyte differentiation through Sox2 and Sox 9 targeting [66] and to the modulation of EAAT-2 expression in astrocytes [67], which is consistent with the role of glutamate excitotoxicity in ALS pathogenesis. Given the putative role of inflammation in ALS pathogenesis, these works may provide new hints into the therapeutic modulation of microglia activation and astrocyte functioning using miRNA-containing exosomes.

Given the involvement of astrocytes in the pathogenesis of SOD1-related ALS, miRNA profile in exosomes released lateral sclerosis (ALS) affected patients, providing diagnostic and prognostic biomarkers. After their purification from patients' biofluids, EVs may be manipulated to accommodate proteins, non-coding RNAs or drugs, and subsequently transferred back to the same patients, serving as therapeutic vehicles

by $S O D 1$-mutated astrocytes has been investigated, despite no significant difference has been found compared to wildtype $S O D 1$ cells [68]. Conversely, EVs released by astrocytes derived from ALS patients carrying C9orf72 HRE present decreased levels of miR-494-3p, a negative regulator of proteins involved in axonal maintenance [69]. Restoration of miR-494-3p levels increases survival in MNs, suggesting that this miRNA may represent a promising therapeutic target [69].

Additionally, recent researches have investigated the utility of circulating miRNAs in EVs derived from ALS patients' blood as potential biomarkers.

MiR-27a-3p, a miRNA involved in myoblast-osteoblast interaction, was detected at decreased concentrations in exosomes derived from ALS serum in comparison to healthy controls [70]. To obtain an ALS-associated miRNA signature, Saucier and colleagues have used a next-generation sequencing approach on EVs extracted from plasma of patients with ALS respect to healthy controls [71]. Differential expression of 22 miRNAs has been observed between ALS and controls and deregulation of miRNAs with relevance for ALS (i.e. miR-9-5p, miR-183-5p, miR-338-3p, miR-1246) has been identified [71]. Interestingly, miR15a-5p and miR-193a-5p have been linked to ALS diagnosis 
and progression, respectively [71]. Similarly, neuronalderived EVs extracted from plasma of ALS patients have shown deregulation of 30 miRNAs compared to healthy controls [72]. Deregulated miRNAs were involved in synaptic vesicle-related pathways and among these, 4 miRNAs have been similarly deregulated in motor cortex samples of ALS patients [72]. Using an analogous approach, a recent work identified a potential miRNA fingerprint in neural-enriched EVs: 5 miRNAs (miR-146a-5p; miR-199a-3p; miR-151a-3p; miR-151a-5p; miR-199a-5p) showed an up-regulation in ALS samples, while 3 miRNAs (miR-4454; miR-10b-5p; miR-29b-3p) were found to be downregulated in ALS compared to healthy controls [73]. Of note, dysregulation of miR-199a-3p and miR-4454 was identified also by Saucier et al. [71] and miR-199a-3p was associated to cell growth, axonal regeneration and plasticity. Moreover, miR-146a-5p, a miRNA involved in synaptic plasticity and inflammatory response, showed reduced expression in CSF of patients with ALS [74] and seems to play a relevant role in Spinal Muscular Atrophy (SMA) as well [75]. As previously mentioned, miR-146a-5p-containing EVs are able to reduce dendritic spine density and miniature synaptic currents in rats, due to inhibition of synaptotagmin 1 and neuroligin 1 [35]. MiR-151a-5p, which is linked to cell viability and oxidative stress response, has shown increased expression in blood and CSF samples from patients with AD [76] and PD [77].

Finally, it is worth mentioning that blood levels of miR151a-5p, miR199a-5p, miR199a-3p, identified as upregulated in ALS by Banack and colleagues, have shown a significant correlation with clinical parameters [78].

Although still at infancy, these studies have opened the path for the utilization of EVs as elegant and innovative tools to study non-coding RNAs, possibly informative in ALS pathogenesis and diagnosis. Since the main function of miRNAs is to downregulate mRNA expression through translation block or transcript degradation, it is reasonable that recently identified miRNAs will add piece of information about disease-related genes and pathways. So far, only one study has successfully performed a RNA sequencing of mRNAs from CSF exosomes in ALS patients, identifying several candidate genes playing a role in processes related to ALS pathogenesis, such as unfolded protein response, ubiquitin-proteasome system and oxidative stress [79].

\section{Clinical application of EVs in ALS}

\section{EVs as biomarkers of diagnosis and prognosis}

Despite several research efforts in elucidating disease mechanisms, ALS pathogenesis is still not completely understood. Moreover, a number of experimental therapeutic approaches have been tested so far, without successful results. Non-invasive measurable and reliable biomarkers are urgently needed to achieve earlier patient diagnosis and inclusion in clinical trial, prognostic estimation and drug monitoring [80].

CNS-derived exosomes have been demonstrated to cross the BBB and enter the systemic circulation, being easily detectable in biological fluids like blood, CSF and urine. They carry specific molecular signatures, reflecting the cell status and functioning during disease condition, thus providing precious information about disease pathogenesis. Moreover, they can be easily isolated from patients' blood and CSF through a minimally invasive maneuver [81], serving as sources of potential biomarkers in neurodegenerative disorders, including ALS.

A few studies have investigated the feasibility of this approach in biofluids from patients with ALS (Table 1). A recent work aimed to characterize number, size and content of EVs retrieved from plasma has found increased mean size in ALS patients compared to healthy controls, but no numerical difference between the two groups [82]. MVs but not exosomes of ALS patients were enriched in ALS-related proteins, including SOD1, TDP-43, pTDP-43 and FUS, confirming the findings of in vitro studies in support of EVmediated prion-like propagation of ALS disease [82]. Similarly, TDP-43 has been retrieved in brain-derived exosomes from CSF of ALS patients, possibly reflecting in vivo the underlying neuropathological findings [54]. Moreover, leukocyte-derived MVs have been found at elevated levels in ALS patients and have shown a slight correlation with progression rate at the last visit, possibly representing biomarkers of disease progression [83].

Besides, analyses of patients' biofluids have drawn the attention on new proteins possibly involved in ALS pathogenesis. Consistent with the role of inflammation in ALS, Chen and colleagues have found elevated levels of interleukin-6 (IL-6) in astrocyte-derived exosomes retrieved from plasma of sALS patients, in addition to a positive correlation between their levels and rate of disease progression [84]. A proteomic study performed on exosome-enriched fraction of CSF has revealed increased concentration of novel INHAT repressor (NIR), involved in nucleolar function, in ALS patients [85]; interestingly NIR has shown altered subcellular localization in MNs of affected patients.

\section{EVs as therapeutic tools}

Other than helpful promising biomarkers, exosomes, and EVs overall, can be employed as potential therapeutics, either as targets or as vehicles of molecules relevant for cell functioning and crosstalk.

EVs are putative carriers of pathological misfolded proteins in neurodegenerative diseases; thereby, attenuating their functions might be beneficial to hamper the seed 
transmission and to prevent the development of neurodegeneration. In this context, several approaches targeting different stages of vesicles' pathways could be exploited, including vesicle formation, release, trafficking and uptake [86]. These strategies have been already applied in inflammation and cancer, but their application in the field of neurodegenerative disorders should be taken into account.

The use of EVs as efficient systems to deliver molecules (e.g. proteins, genetic material or drugs) targeting cells or tissues likely represents the most innovative field of application. There are several advantages of using EVs as carriers of relevant biological molecules. First, they are highly stable in circulating system, in a way that can reach also distant targets; moreover, exosomes can cross the BBB [3] enabling the transport of cargoes to the CNS, being particularly appealing for the treatment of neurologic disorders. Then, compared to liposome- and nano-based therapies, they have cell-surface molecules and high affinity for tissues, reducing the risk of off-target effects, as well as avoiding phagocytosis or degradation by macrophages. In addition to their excellent biocompatibility and poor immunogenicity and toxicity, EVs show high availability, since they can easily be retrieved from patients' biofluids. Ideally, after isolating exosomes from cell cultures or patients' blood, they can be manipulated to accommodate proteins, non-coding RNAs or drugs, and subsequently transferred back to the same patients (Fig. 1).

It is worth mentioning that stem cells secrete exosomes containing cell-derived factors, which, through a paracrine effect, can modulate the surrounding biological environment. Mesenchymal stem cells (MSCs) can be easily isolated from adult and fetal tissues, including adipose tissue, and their several beneficial effects (tissue homeostasis, repair and regeneration) are at least partially mediated by exosome secretion [6]. Exosomes derived from human mesenchymal stem cells selectively promoted neurite outgrowth in cortical neuron cultures [87]. This strategy has been considered extremely attractive and valuable in the treatment of neurodegenerative disorders, including ALS.

At this purpose, exosomes isolated from murine ASCs have been shown to protect wild-type and SOD1-mutated MN-like NSC-34 cells from oxidative stress, increasing cell viability [88]. Similarly, they had the ability of restoring complex I activity, coupling efficiency and mitochondrial membrane potential [89]. Proteomic analysis performed on ASC-derived exosomes confirmed their putative neuroprotective role, revealing the expression of proteins involved in cell adhesion and negative regulation of apoptosis [38]. Moreover, ASC-derived exosomes were effective in reducing aggregation of SOD1 and modulating mitochondrial dysfunction in neuronal cells from SOD1 mouse models [90]. In parallel, the use of ASCs and their exosomes as therapeutic tools in ALS has been validated in vivo as well. Systemic administration of ASCs in SOD1-mutated mouse delayed motor deterioration, increased $\mathrm{MN}$ number and neurotrophin levels into murine spinal cord [91]. A recent study demonstrated that repeated administrations of ASC-derived exosomes improved motor performance in SOD1-mutated mouse, protected spinal MNs from neurodegeneration, preserved neuromuscular junction, muscle morphology and functioning, and reduced glial cell activation [92]. Additionally, intranasally delivered exosomes were able to reach brains of wild-type mice and to home to injured brain regions of SOD1-mutated counterparts.

However, there are also some limitations hampering the translation of EVs in clinical therapies, which are mainly related to the challenge of purifying the scarce number of exosomes from the human body and of determining the best source for their retrieval. In addition, the optimization of vesicle loading and dosage is equally important to maintain the structure and the content of exosomal membranes without altering functional efficacy. Finally, the problem of how to reach selectively the target cells must be addressed.

\section{Concluding remarks}

The development and the spreading of ALS and other neurodegenerative disorders have been linked to the cell-to-cell propagation of misfolded proteins in a prion-like fashion. Although a wide body of evidence supporting the role of EVs in this process in ALS, in vivo studies are still poor. Further, there is also contrasting evidence pointing at a beneficial role of EVs in disease pathogenesis. Nonetheless, EVs have undoubtedly added a piece of knowledge in understanding disease pathomechanisms. Moreover, the isolation of peripheral and brain-derived EVs from patients' biofluids have provided valuable insights into their clinical application as diagnostic and prognostic biomarkers, enabling the study in vivo of molecular and pathological correlates of ALS. Despite not being so close to their use as therapeutics in the oncoming futures, the findings fulfilled so far are promising and should encourage further research efforts in this direction.

Acknowledgments We gratefully thank the Associazione Centro Dino Ferrari for its support. Regione Lombardia TRANS-ALS and RF-201602362317/Italian Ministry of Health to GPC is gratefully acknowledged. Italian Ministry of health "RF-2018-12366357" grant to SC is gratefully acknowledged.

Author contributions DG: conceptualization, literature search and data analysis, writing - original draft preparation, visualization. NB and GPC: writing — review and editing; SC: supervision, writing — review and editing. All authors have read and agreed to the published version of the manuscript. 
Funding Open access funding provided by Università degli Studi di Milano within the CRUI-CARE Agreement. This work was partially supported by a grant from the Ministero della Salute Fondazione IRCCS Ca' Granda Ospedale Maggiore Policlinico, Ricerca Corrente 2020 .

\section{Compliance with ethical standards}

Conflict of interest The authors declare no conflict of interest.

Open Access This article is licensed under a Creative Commons Attribution 4.0 International License, which permits use, sharing, adaptation, distribution and reproduction in any medium or format, as long as you give appropriate credit to the original author(s) and the source, provide a link to the Creative Commons licence, and indicate if changes were made. The images or other third party material in this article are included in the article's Creative Commons licence, unless indicated otherwise in a credit line to the material. If material is not included in the article's Creative Commons licence and your intended use is not permitted by statutory regulation or exceeds the permitted use, you will need to obtain permission directly from the copyright holder. To view a copy of this licence, visit http://creativecommons.org/licenses/by/4.0/.

\section{References}

1. Akers JC, Gonda D, Kim R et al (2013) Biogenesis of extracellular vesicles $(\mathrm{EV})$ : exosomes, microvesicles, retrovirus-like vesicles, and apoptotic bodies. J Neurooncol 113:1-11. https://doi. org/10.1007/s11060-013-1084-8

2. Cocucci E, Meldolesi J (2015) Ectosomes and exosomes: shedding the confusion between extracellular vesicles. Trends Cell Biol 25:364-372. https://doi.org/10.1016/j.tcb.2015.01.004

3. Matsumoto J, Stewart T, Banks WA, Zhang J (2017) The transport mechanism of extracellular vesicles at the blood-brain barrier. Curr Pharm Des 23:6206-6214. https://doi.org/10.2174/13816 12823666170913164738

4. Kourembanas S (2015) Exosomes: vehicles of intercellular signaling, biomarkers, and vectors of cell therapy. Annu Rev Physiol 77:13-27. https://doi.org/10.1146/annurev-physiol-021014-07164 1

5. Théry C, Amigorena S, Raposo G, Clayton A (2006) Isolation and characterization of exosomes from cell culture supernatants and biological fluids. Curr Protocol Cell Biol 30:3.22.1-3.22.29. https://doi.org/10.1002/0471143030.cb0322s30

6. Lai RC, Yeo RWY, Lim SK (2015) Mesenchymal stem cell exosomes. Semin Cell Dev Biol 40:82-88. https://doi. org/10.1016/j.semcdb.2015.03.001

7. Greco V, Hannus M, Eaton S (2001) Argosomes: a potential vehicle for the spread of morphogens through epithelia. Cell 106:633645. https://doi.org/10.1016/S0092-8674(01)00484-6

8. Turturici G, Tinnirello R, Sconzo G, Geraci F (2014) Extracellular membrane vesicles as a mechanism of cell-to-cell communication: advantages and disadvantages. Am J Physiol Cell Physiol 306:C621-C633. https://doi.org/10.1152/ajpcell.00228.2013

9. An K, Klyubin I, Kim Y et al (2013) Exosomes neutralize synaptic-plasticity-disrupting activity of $\mathrm{A} \beta$ assemblies in vivo. Mol Brain 6:47. https://doi.org/10.1186/1756-6606-6-47

10. Valadi H, Ekström K, Bossios A et al (2007) Exosome-mediated transfer of mRNAs and microRNAs is a novel mechanism of genetic exchange between cells. Nat Cell Biol 9:654-659. https:// doi.org/10.1038/ncb1596
11. Basso M, Bonetto V (2016) Extracellular vesicles and a novel form of communication in the brain. Front Neurosci 10:127. https ://doi.org/10.3389/fnins.2016.00127

12. Fauré J, Lachenal G, Court M et al (2006) Exosomes are released by cultured cortical neurones. Mol Cell Neurosci 31:642-648. https://doi.org/10.1016/j.mcn.2005.12.003

13. Lachenal G, Pernet-Gallay K, Chivet M et al (2011) Release of exosomes from differentiated neurons and its regulation by synaptic glutamatergic activity. Mol Cell Neurosci 46:409-418. https ://doi.org/10.1016/j.men.2010.11.004

14. Antonucci F, Turola E, Riganti L et al (2012) Microvesicles released from microglia stimulate synaptic activity via enhanced sphingolipid metabolism. EMBO J 31:1231-1240. https://doi. org/10.1038/emboj.2011.489

15. Thompson AG, Gray E, Heman-Ackah SM et al (2016) Extracellular vesicles in neurodegenerative disease-pathogenesis to biomarkers. Nat Rev Neurol 12:346-357. https://doi. org/10.1038/nrneurol.2016.68

16. Fröhlich D, Kuo WP, Frühbeis C et al (2014) Multifaceted effects of oligodendroglial exosomes on neurons: impact on neuronal firing rate, signal transduction and gene regulation. Philos Trans R Soc Lond B Biol Sci 369(1652):20130510. https ://doi.org/10.1098/rstb.2013.0510

17. Upadhya R, Zingg W, Shetty S, Shetty AK (2020) Astrocytederived extracellular vesicles: neuroreparative properties and role in the pathogenesis of neurodegenerative disorders. J Control Release 323:225-239. https://doi.org/10.1016/j.jconr el.2020.04.017

18. Gosselin R-D, Meylan P, Decosterd I (2013) Extracellular microvesicles from astrocytes contain functional glutamate transporters: regulation by protein kinase $\mathrm{C}$ and cell activation. Front Cell Neurosci 7:251. https://doi.org/10.3389/fncel .2013.00251

19. Eitan E, Green J, Bodogai M et al (2017) Age-related changes in plasma extracellular vesicle characteristics and internalization by leukocytes. Sci Rep 7:1342. https://doi.org/10.1038/s4159 8-017-01386-z

20. Goedert M, Clavaguera F, Tolnay M (2010) The propagation of prion-like protein inclusions in neurodegenerative diseases. Trends Neurosci 33:317-325. https://doi.org/10.1016/j. tins.2010.04.003

21. Walsh DM, Selkoe DJ (2016) A critical appraisal of the pathogenic protein spread hypothesis of neurodegeneration. Nat Rev Neurosci 17:251-260. https://doi.org/10.1038/nrn.2016.13

22. Peng C, Trojanowski JQ, Lee VM-Y (2020) Protein transmission in neurodegenerative disease. Nat Rev Neurol 16:199-212. https ://doi.org/10.1038/s41582-020-0333-7

23. Magalhães AC, Baron GS, Lee KS et al (2005) Uptake and neuritic transport of scrapie prion protein coincident with infection of neuronal cells. J Neurosci 25:5207-5216. https://doi. org/10.1523/JNEUROSCI.0653-05.2005

24. Gousset K, Schiff E, Langevin C et al (2009) Prions hijack tunnelling nanotubes for intercellular spread. Nat Cell Biol 11:328336. https://doi.org/10.1038/ncb1841

25. Asai H, Ikezu S, Tsunoda S et al (2015) Depletion of microglia and inhibition of exosome synthesis halt tau propagation. Nat Neurosci 18:1584-1593. https://doi.org/10.1038/nn.4132

26. Emmanouilidou E, Melachroinou K, Roumeliotis $\mathrm{T}$ et al (2010) Cell-produced alpha-synuclein is secreted in a calciumdependent manner by exosomes and impacts neuronal survival. J Neurosci 30:6838-6851. https://doi.org/10.1523/JNEUR OSCI.5699-09.2010

27. Ngolab J, Trinh I, Rockenstein E et al (2017) Brain-derived exosomes from dementia with Lewy bodies propagate $\alpha$-synuclein pathology. Acta Neuropathol Commun 5:46. https ://doi.org/10.1186/s40478-017-0445-5 
28. Perez-Gonzalez R, Gauthier SA, Kumar A, Levy E (2012) The exosome secretory pathway transports amyloid precursor protein carboxyl-terminal fragments from the cell into the brain extracellular space. J Biol Chem 287:43108-43115. https://doi. org/10.1074/jbc.M112.404467

29. Saman S, Kim W, Raya M et al (2012) Exosome-associated tau is secreted in tauopathy models and is selectively phosphorylated in cerebrospinal fluid in early Alzheimer disease. J Biol Chem 287:3842-3849. https://doi.org/10.1074/jbc.M111.27706 1

30. Shi M, Liu C, Cook TJ et al (2014) Plasma exosomal $\alpha$-synuclein is likely CNS-derived and increased in Parkinson's disease. Acta Neuropathol 128:639-650. https://doi.org/10.1007/s0040 1-014-1314-y

31. Wang Y, Balaji V, Kaniyappan S et al (2017) The release and trans-synaptic transmission of Tau via exosomes. Mol Neurodegener 12(1):5. https://doi.org/10.1186/s13024-016-0143-y

32. Kfoury N, Holmes BB, Jiang H et al (2012) Trans-cellular propagation of Tau aggregation by fibrillar species. J Biol Chem 287:19440-19451. https://doi.org/10.1074/jbc.M112.346072

33. Garden GA, La Spada AR (2012) Intercellular (mis)communication in neurodegenerative disease. Neuron 73:886-901. https:// doi.org/10.1016/j.neuron.2012.02.017

34. Paolicelli RC, Bergamini G, Rajendran L (2019) Cell-to-cell communication by extracellular vesicles: focus on microglia. Neuroscience 405:148-157. https://doi.org/10.1016/j.neuroscien ce.2018.04.003

35. Prada I, Gabrielli M, Turola E et al (2018) Glia-to-neuron transfer of miRNAs via extracellular vesicles: a new mechanism underlying inflammation-induced synaptic alterations. Acta Neuropathol 135:529-550. https://doi.org/10.1007/s00401-017-1803-x

36. Counil H, Krantic S (2020) Synaptic activity and (neuro)inflammation in Alzheimer's disease: could exosomes be an additional link? J Alzheimers Dis 74:1029-1043. https://doi.org/10.3233/ JAD-191237

37. Marchetti B, Leggio L, L'Episcopo F et al (2020) Glia-derived extracellular vesicles in Parkinson's disease. J Clin Med 9:1941. https://doi.org/10.3390/jcm9061941

38. Bonafede R, Brandi J, Manfredi M et al (2019) The anti-apoptotic effect of ASC-exosomes in an in vitro ALS model and their proteomic analysis. Cells 8:1087. https://doi.org/10.3390/cells80910 87

39. Neumann M, Sampathu DM, Kwong LK et al (2006) Ubiquitinated TDP-43 in frontotemporal lobar degeneration and amyotrophic lateral sclerosis. Science 314:130-133. https://doi. org/10.1126/science. 1134108

40. Silverman JM, Fernando SM, Grad LI et al (2016) Disease mechanisms in ALS: misfolded SOD1 transferred through exosomedependent and exosome-independent pathways. Cell Mol Neurobiol 36:377-381. https://doi.org/10.1007/s10571-015-0294-3

41. Forsberg K, Andersen PM, Marklund SL, Brännström T (2011) Glial nuclear aggregates of superoxide dismutase-1 are regularly present in patients with amyotrophic lateral sclerosis. Acta Neuropathol 121:623-634. https://doi.org/10.1007/s00401-011-0805-3

42. Saberi S, Stauffer JE, Schulte DJ, Ravits J (2015) Neuropathology of amyotrophic lateral sclerosis and its variants. Neurol Clin 33:855-876. https://doi.org/10.1016/j.ncl.2015.07.012

43. Gomes C, Keller S, Altevogt P, Costa J (2007) Evidence for secretion of $\mathrm{Cu}, \mathrm{Zn}$ superoxide dismutase via exosomes from a cell model of amyotrophic lateral sclerosis. Neurosci Lett 428:43-46. https://doi.org/10.1016/j.neulet.2007.09.024

44. Basso M, Pozzi S, Tortarolo M et al (2013) Mutant copper-zinc superoxide dismutase (SOD1) induces protein secretion pathway alterations and exosome release in astrocytes implications for disease spreading and motor neuron pathology in amyotrophic lateral sclerosis. J Biol Chem 288:15699-15711. https://doi. org/10.1074/jbc.M112.425066

45. Grad LI, Guest WC, Yanai A et al (2011) Intermolecular transmission of superoxide dismutase 1 misfolding in living cells. Proc Natl Acad Sci U S A 108:16398-16403. https://doi.org/10.1073/ pnas. 1102645108

46. Münch C, O'Brien J, Bertolotti A (2011) Prion-like propagation of mutant superoxide dismutase-1 misfolding in neuronal cells. Proc Natl Acad Sci U S A 108:3548-3553. https://doi.org/10.1073/ pnas. 1017275108

47. Grad LI, Yerbury JJ, Turner BJ et al (2014) Intercellular propagated misfolding of wild-type $\mathrm{Cu} / \mathrm{Zn}$ superoxide dismutase occurs via exosome-dependent and -independent mechanisms. Proc Natl Acad Sci U S A 111:3620-3625. https://doi.org/10.1073/ pnas. 1312245111

48. Massenzio F, Peña-Altamira E, Petralla $S$ et al (2018) Microglial overexpression of fALS-linked mutant SOD1 induces SOD1 processing impairment, activation and neurotoxicity and is counteracted by the autophagy inducer trehalose. Biochim Biophys Acta Mol Basis Dis 1864:3771-3785. https://doi. org/10.1016/j.bbadis.2018.10.013

49. Silverman JM, Christy D, Shyu CC et al (2019) CNS-derived extracellular vesicles from superoxide dismutase 1 (SOD1) G93A ALS mice originate from astrocytes and neurons and carry misfolded SOD1. J Biol Chem 294:3744-3759. https:// doi.org/10.1074/jbc.RA118.004825

50. Nonaka T, Masuda-Suzukake M, Arai T et al (2013) Prion-like properties of pathological TDP-43 aggregates from diseased brains. Cell Rep 4:124-134. https://doi.org/10.1016/j.celrep.2013.06.007

51. Rutherford NJ, Zhang Y-J, Baker M et al (2008) Novel mutations in TARDBP (TDP-43) in patients with familial amyotrophic lateral sclerosis. PLoS Genet 4:e1000193. https://doi. org/10.1371/journal.pgen.1000193

52. Nonaka T, Kametani F, Arai T et al (2009) Truncation and pathogenic mutations facilitate the formation of intracellular aggregates of TDP-43. Hum Mol Genet 18:3353-3364. https:// doi.org/10.1093/hmg/ddp275

53. Ding X, Ma M, Teng J et al (2015) Exposure to ALS-FTDCSF generates TDP-43 aggregates in glioblastoma cells through exosomes and TNTs-like structure. Oncotarget 6:24178-24191. https://doi.org/10.18632/oncotarget.4680

54. Feneberg E, Steinacker P, Lehnert S et al (2014) Limited role of free TDP-43 as a diagnostic tool in neurodegenerative diseases. Amyotroph Lateral Scler Frontotemporal Degener 15:351-356. https://doi.org/10.3109/21678421.2014.905606

55. Feiler MS, Strobel B, Freischmidt A et al (2015) TDP-43 is intercellularly transmitted across axon terminals. J Cell Biol 211:897-911. https://doi.org/10.1083/jcb.201504057

56. Iguchi Y, Eid L, Parent $M$ et al (2016) Exosome secretion is a key pathway for clearance of pathological TDP-43. Brain 139:3187-3201. https://doi.org/10.1093/brain/aww237

57. Mackenzie IR, Rademakers R, Neumann M (2010) TDP-43 and FUS in amyotrophic lateral sclerosis and frontotemporal dementia. Lancet Neurol 9:995-1007. https://doi.org/10.1016/S1474 $-4422(10) 70195-2$

58. Kamelgarn M, Chen J, Kuang L et al (2016) Proteomic analysis of FUS interacting proteins provides insights into FUS function and its role in ALS. Biochim Biophys Acta Mol Basis Dis 1862:2004-2014. https://doi.org/10.1016/j.bbadis.2016.07.015

59. Ash PEA, Bieniek KF, Gendron TF et al (2013) Unconventional translation of C9ORF72 GGGGCC expansion generates insoluble polypeptides specific to c9FTD/ALS. Neuron 77:639-646. https://doi.org/10.1016/j.neuron.2013.02.004 
60. Wen X, Tan W, Westergard T et al (2014) Antisense prolinearginine RAN dipeptides linked to C9ORF72-ALS/FTD form toxic nuclear aggregates that initiate in vitro and in vivo neuronal death. Neuron 84:1213-1225. https://doi.org/10.1016/j. neuron.2014.12.010

61. Westergard T, Jensen BK, Wen X et al (2016) Cell-to-cell transmission of dipeptide repeat proteins linked to C9orf72-ALS/ FTD. Cell Rep 17:645-652. https://doi.org/10.1016/j.celre p.2016.09.032

62. Haramati S, Chapnik E, Sztainberg Y et al (2010) miRNA malfunction causes spinal motor neuron disease. Proc Natl Acad Sci U S A 107:13111-13116. https://doi.org/10.1073/pnas.10061 51107

63. Gagliardi D, Comi GP, Bresolin N, Corti S (2019) MicroRNAs as regulators of cell death mechanisms in amyotrophic lateral sclerosis. J Cell Mol Med 23:1647-1656. https://doi. org/10.1111/jcmm. 13976

64. Hosaka T, Yamashita T, Tamaoka A, Kwak S (2019) Extracellular RNAs as biomarkers of sporadic amyotrophic lateral sclerosis and other neurodegenerative diseases. Int J Mol Sci 20:3148. https://doi.org/10.3390/ijms20133148

65. Pinto S, Cunha C, Barbosa M et al (2017) Exosomes from NSC-34 cells transfected with hSOD1-G93A are enriched in miR-124 and drive alterations in microglia phenotype. Front Neurosci 11:273. https://doi.org/10.3389/fnins.2017.00273

66. Zhou F, Guan Y, Chen Y et al (2013) miRNA-9 expression is upregulated in the spinal cord of G93A-SOD1 transgenic mice. Int J Clin Exp Pathol 6:1826-1838

67. Morel L, Regan M, Higashimori H et al (2013) Neuronal exosomal miRNA-dependent translational regulation of astroglial glutamate transporter GLT1. J Biol Chem 288:7105-7116. https ://doi.org/10.1074/jbc.M112.410944

68. Jovičić A, Gitler AD (2017) Distinct repertoires of microRNAs present in mouse astrocytes compared to astrocyte-secreted exosomes. PLoS ONE 12:e0171418. https://doi.org/10.1371/journ al.pone. 0171418

69. Varcianna A, Myszczynska MA, Castelli LM et al (2019) MicroRNAs secreted through astrocyte-derived extracellular vesicles cause neuronal network degeneration in C9orf72 ALS. EBioMedicine 40:626-635. https://doi.org/10.1016/j.ebiom.2018.11.067

70. Xu Q, Zhao Y, Zhou X et al (2018) Comparison of the extraction and determination of serum exosome and miRNA in serum and the detection of miR-27a-3p in serum exosome of ALS patients. Intractable Rare Dis Res 7:13-18. https://doi.org/10.5582/ irdr.2017.01091

71. Saucier D, Wajnberg G, Roy J et al (2019) Identification of a circulating miRNA signature in extracellular vesicles collected from amyotrophic lateral sclerosis patients. Brain Res 1708:100-108. https://doi.org/10.1016/j.brainres.2018.12.016

72. Katsu M, Hama Y, Utsumi J et al (2019) MicroRNA expression profiles of neuron-derived extracellular vesicles in plasma from patients with amyotrophic lateral sclerosis. Neurosci Lett 708:134176. https://doi.org/10.1016/j.neulet.2019.03.048

73. Banack SA, Dunlop RA, Cox PA (2020) An miRNA fingerprint using neural-enriched extracellular vesicles from blood plasma: towards a biomarker for amyotrophic lateral sclerosis/motor neuron disease. Open Biol 10:200116. https://doi.org/10.1098/ rsob. 200116

74. Waller R, Wyles M, Heath PR et al (2017) Small RNA sequencing of sporadic amyotrophic lateral sclerosis cerebrospinal fluid reveals differentially expressed miRNAs related to neural and Glial activity. Front Neurosci 11:731. https://doi.org/10.3389/ fnins.2017.00731

75. Sison SL, Patitucci TN, Seminary ER et al (2017) Astrocyteproduced miR-146a as a mediator of motor neuron loss in spinal muscular atrophy. Hum Mol Genet 26:3409-3420. https://doi. org/10.1093/hmg/ddx230

76. Leidinger P, Backes C, Deutscher S et al (2013) A blood based 12-miRNA signature of Alzheimer disease patients. Genome Biol 14:R78. https://doi.org/10.1186/gb-2013-14-7-r78

77. dos Santos MCT, Barreto-Sanz MA, Correia BRS et al (2018) miRNA-based signatures in cerebrospinal fluid as potential diagnostic tools for early stage Parkinson's disease. Oncotarget 9:17455-17465. https://doi.org/10.18632/oncotarget.24736

78. Raheja R, Regev K, Healy BC et al (2018) Correlating serum micrornas and clinical parameters in amyotrophic lateral sclerosis. Muscle Nerve 58:261-269. https://doi.org/10.1002/mus.26106

79. Otake K, Kamiguchi H, Hirozane Y (2019) Identification of biomarkers for amyotrophic lateral sclerosis by comprehensive analysis of exosomal mRNAs in human cerebrospinal fluid. BMC Med Genomics 12:7. https://doi.org/10.1186/s12920-019-0473-z

80. Gagliardi D, Meneri M, Saccomanno D et al (2019) Diagnostic and prognostic role of blood and cerebrospinal fluid and blood neurofilaments in amyotrophic lateral sclerosis: a review of the literature. Int J Mol Sci 20(17):4152. https://doi.org/10.3390/ijms2 0174152

81. Hornung S, Dutta S, Bitan G (2020) CNS-derived blood exosomes as a promising source of biomarkers: opportunities and challenges. Front Mol Neurosci 13:38. https://doi.org/10.3389/fnmol .2020 .00038

82. Sproviero D, La Salvia S, Giannini M et al (2018) Pathological proteins are transported by extracellular vesicles of sporadic amyotrophic lateral sclerosis patients. Front Neurosci 12:487. https://doi.org/10.3389/fnins.2018.00487

83. Sproviero D, La Salvia S, Colombo F et al (2019) Leukocyte derived microvesicles as disease progression biomarkers in slow progressing amyotrophic lateral sclerosis patients. Front Neurosci 13:344. https://doi.org/10.3389/fnins.2019.00344

84. Chen Y, Xia K, Chen L, Fan D (2019) Increased interleukin-6 levels in the astrocyte-derived exosomes of sporadic amyotrophic lateral sclerosis patients. Front Neurosci 13:574. https://doi. org/10.3389/fnins.2019.00574

85. Hayashi N, Doi H, Kurata Y et al (2019) Proteomic analysis of exosome-enriched fractions derived from cerebrospinal fluid of amyotrophic lateral sclerosis patients. Neurosci Res. https://doi. org/10.1016/j.neures.2019.10.010

86. Samanta S, Rajasingh S, Drosos N et al (2018) Exosomes: new molecular targets of diseases. Acta Pharmacol Sin 39:501-513. https://doi.org/10.1038/aps.2017.162

87. Lopez-Verrilli MA, Caviedes A, Cabrera A et al (2016) Mesenchymal stem cell-derived exosomes from different sources selectively promote neuritic outgrowth. Neuroscience 320:129-139. https:// doi.org/10.1016/j.neuroscience.2016.01.061

88. Bonafede R, Scambi I, Peroni D et al (2016) Exosome derived from murine adipose-derived stromal cells: neuroprotective effect on in vitro model of amyotrophic lateral sclerosis. Exp Cell Res 340:150-158. https://doi.org/10.1016/j.yexcr.2015.12.009

89. Calabria E, Scambi I, Bonafede R et al (2019) ASCs-exosomes recover coupling efficiency and mitochondrial membrane potential in an in vitro model of ALS. Front Neurosci 13:1070. https://doi. org/10.3389/fnins.2019.01070

90. Lee M, Ban J-J, Kim KY et al (2016) Adipose-derived stem cell exosomes alleviate pathology of amyotrophic lateral sclerosis in vitro. Biochem Biophys Res Commun 479:434-439. https:// doi.org/10.1016/j.bbrc.2016.09.069

91. Marconi S, Bonaconsa M, Scambi I et al (2013) Systemic treatment with adipose-derived mesenchymal stem cells ameliorates clinical and pathological features in the amyotrophic lateral sclerosis murine model. Neuroscience 248:333-343. https://doi. org/10.1016/j.neuroscience.2013.05.034 
92. Bonafede R, Turano E, Scambi I et al (2020) ASC-exosomes ameliorate the disease progression in SOD1(G93A) murine model underlining their potential therapeutic use in human ALS. Int $\mathbf{J}$ Mol Sci 21(10):3651. https://doi.org/10.3390/ijms21103651
Publisher's Note Springer Nature remains neutral with regard to jurisdictional claims in published maps and institutional affiliations. 